\title{
Taming the RAGE of Alzheimer's disease
}

The accumulation of amyloid- $\beta$ $(\mathrm{A} \beta)$ in the brain of patients with Alzheimer's disease (AD) is hypothesized to initiate a cascade of events culminating in neuronal loss and dementia. However, therapeutic approaches designed to reduce the production or aggregation of $A \beta$ in the brain have so far failed in the clinic. Now, a study published in Journal of Clinical Investigation reports a novel small-molecule inhibitor of the receptor for advanced glycation end products (RAGE), which safely reduces $A \beta$ levels in the brain and normalizes cognitive performance in an aged mouse model of AD.

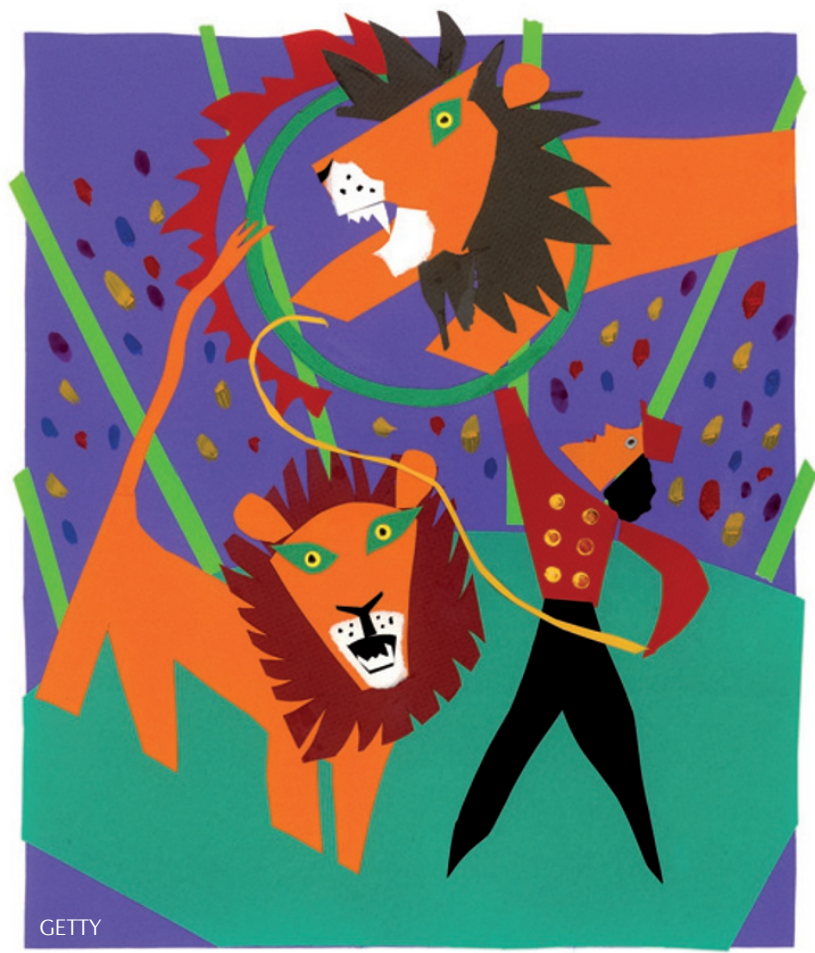

RAGE is a member of the immunoglobulin superfamily, which binds multiple ligands through its extracellular V domain. In AD, RAGE acts as a cell surface receptor for $A \beta$ at the blood-brain barrier $(\mathrm{BBB})$ and in neurons and microglia, contributing to neurodegeneration via multiple pathways. Although preclinical studies implicate RAGE as an important therapeutic target in $\mathrm{AD}$, anti-RAGE therapy has yet to be successfully developed. Indeed, although an azole-based firstgeneration RAGE inhibitor entered a Phase II trial in patients with $\mathrm{AD}$, it was terminated - probably owing to toxicity. Deane and colleagues therefore set out to identify a novel high-affinity A $\beta$-RAGE blocker that was safe and non-toxic.

First, the authors screened a diverse library of 5,000 small organic compounds, and identified three new tertiary amides that were capable of blocking the $A \beta-$ RAGE interaction. Based on the common structural features of these lead molecules, they synthesized a second-generation library of compounds, from which they identified a high-affinity RAGE-specific inhibitor that could penetrate the BBB: FPS-ZM1. In vitro, FPS-ZM1 specifically blocked $\mathrm{A} \beta$ binding to the $\mathrm{V}$ domain of RAGE, thus inhibiting $A \beta$-mediated cellular stress.

Next, Deane and colleagues investigated the effects of their compound in vivo. In 15- to 17-month-old $A P P^{s w / 0}$ mice (a transgenic mouse model of AD that overexpresses human amyloid precursor protein (APP)), FPS-ZM1 almost completely inhibited the RAGE-mediated transport of circulating $A \beta$ across the BBB. Further studies showed that after crossing the BBB, FPS-ZM1 bound exclusively to RAGE, also blocking the actions of RAGE within the brain. Indeed, intraperitoneal administration of FPS-ZM1 (1 mg per $\mathrm{kg}$ ) to aged $A P P^{\mathrm{s} w / 0}$ mice daily for 2 months reduced the activity of nuclear factor- $\kappa \mathrm{B}$ and the $\beta$-secretase enzyme BACE1 in neural cells, thereby blocking $A \beta$ production. This effect, in combination with the inhibition of $A \beta$ influx into the brain, resulted in a $60-80 \%$ reduction in levels of $A \beta 40$ and $A \beta 42$ in the cortex and hippocampus, respectively. Furthermore, FPS-ZM1 completely restored cerebral blood flow and behavioural responses, and markedly suppressed activation of microglia and the neuroinflammatory responses in these mice. Importantly, there were no signs of toxicity, even at a dose 500-fold higher than the therapeutic dose used.

These findings provide further support for the concept of RAGE inhibition as a therapeutic approach for the treatment of $\mathrm{AD}$, and identify FPS-ZM1 as a promising lead compound.

Sarah Crunkhorn

ORIGINAL RESEARCH PAPER Deane, R. et al. A multimodal RAGE-specific inhibitor reduces amyloid $\beta$-mediated brain disorder in a mouse model of Alzheimer disease. J. Clin. Invest. 122, 1377-1392 (2012) 